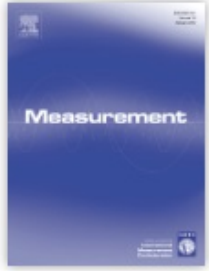

ISSN: $0263-2241$

\section{Submit Your Paper}

\section{View Articles}

\section{Guide for Authors}

$\checkmark$

Abstracting/ Indexing

Track Your Paper

\section{Order Journal}

Sample Issue $\pi$

\section{Journal Metrics \\ CiteScore: $\mathbf{2 . 5 2}$ (1) \\ More about CiteScore \\ Impact Factor: 2.359 (i) \\ 5-Year Impact Factor: 2.255 (i) \\ Source Normalized Impact per Paper (SNIP): 1.630 (1) \\ SCImago Journal Rank (SJR): 0.734 (i) \\ $>$ View More on Journal Insights $\lambda$}

\section{Article Enrichments $\quad \vee$ \\ $>$ AudioSlides \\ $>$ Data in Brief co-submission \\ > Interactive MATLAB Figure Viewer}

Related Links 주

$>$ Author Stats $\pi$ (1)

> Publishing Campus $\pi$

$>$ Author Services

$>$ Try out personalized alert features

\section{Measurement}

Journal of the International Measurement Confederation (IMEKO $x$ )

$>$ Supports Open Access

Editor-in-Chief: K.T.V. Grattan

$>$ View Editorial Board

Contributions are invited on all aspects of the research, development and applications of the science and technology of measurement and

instrumentation. Authors are encouraged to submit novel material which could include results of research or experimental work, may deal with practical developments related...

Read more

\section{Most Downloaded Recent Articles Most Cited Open Access Articles}

Measuring risk-aversion: The challenge $\pi$ P.J. Thomas

Early fault diagnosis of bearing and stator faults of the single-phase induction motor using acoustic signals $\pi$ Adam Glowacz $\mid$ Witold Glowacz $\mid . .$.

Optical flow background estimation for real-time pan/tilt camera object tracking $\pi$ Daniel D. Doyle $\mid$ Alan L. Jennings $\mid \ldots$

View All Articles >

\section{Announcements}

Heliyon Partner Journal त

This journal is now partnering with Heliyon, an open access journal from Elsevier publishing quality peer reviewed research across all disciplines. Partner journals provide authors with an easy route to transfer their research to Heliyon.

>Learn more at Heliyon.com >

$>$ View All

Videos - Audioslides

Videos - Audioslides

Automatic Detection of Onset and Offset of QRS Complexes Independent of Isoelectric Segments

Bearing defect inspection based on machine vision

Measurement and statistical analysis toward reproducibility validation of $\mathrm{AZ}_{45} \mathrm{G}_{2}$ cylindrical microlenses obtained by reflow

\section{$>$ View All}

News

Signal Processing and Control Digital Catalogue 2017

Hear it from the authors: new video commentary!

$>$ View All 
Home > Journals > Measurement > Editorial Board

\section{Submit Your Paper $\quad \checkmark$}

View Articles $\pi$

Guide for Authors

Abstracting/ Indexing

Track Your Paper

Order Journal

Sample Issue $\pi$

Journal Metrics

CiteScore: 2.52 (1)

More about CiteScore

Impact Factor: 2.359

5-Year Impact Factor: 2.255 (1)

Source Normalized Impact per Paper

(SNIP): $1.630 \odot$

SCImago Journal Rank (SJR): 0.734 (

$>$ View More on Journal Insights

Article Enrichments

$>$ Audioslides

Data in Brief co-submission

$>$ Interactive MATLAB Figure Viewer

Related Links

Author Stats $\approx$ (1)

> Publishing Campus ?

$>$ Author Services

$>$ Try out personalized alert features

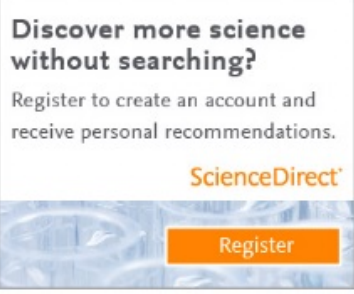

\section{Measurement Editorial Board}

Editor-in-Chief

K.T.V. Grattan

City, University of London, London, UK

Associate Editors

S. Khan

City, University of London, London, England, UK

T. Sun

City, University of London, London, UK

T. Sarkodie-Gyan

University of Texas, El Paso, Texas, USA

A. Lay-Ekuakille

University of Salento, Lecce, Italy

Membership of the Board

S. Bennett

National Physical Laboratory, Teddington, Middlesex, UK

N. Fox

National Physical Laboratory, Teddington, Middlesex, UK

R.Z. Morawski

Warsaw University of Technology, Warsaw, Poland

P.H. Osanna

Technische Universität Wien, Wien, Austria

A. Wallard

National Physical Laboratory, Teddington, Middlesex, UK

Ex-Officio Membership of the Board

G.W. Bahng

Korea Research Institute of Standards and Science, Daejeon, The Republic of Korea

L. Borbas

Budapest University of Technology and Economics, Budapest, Hungary

M. Borsic

Croatian Metrology Society, Zagreb, Croatia

T. Bruns

Physikalisch-Technische Bundesanstalt, Braunschweig, Germany 
P. Daponte

Università degli Studi del Sannio, Benevento, Italy

C. Ferrero

INRIM National Inst. Searches Metrologica, Torino, Italy

J. Holub

Czech Technical University in Prague (Ceské Vysolké Ucení Technické), Prague, Czech Republic

D.I. Kang

KRISS, Taejon, , The Republic of Korea

\section{P. Kneppo}

Czech Technical University in Prague (Ceské Vysoké Ucení Technické), Czech Republic

Y. Koike

Tokyo Institute of Technology, Midori-ku, Yokohama, Japan

\section{R. Kumme}

Physikalisch-Technische Bundesanstalt, Braunschweig, Germany

L. Mari

CETIC, Castellanza (VA), Italy

L. Michaeli

Technical University Kosice, Kosice, Slovakia

L. Monostori

Hungarian Academy of Sciences, Budapest, Hungary

F. Pavese

Istituto Nazionale di Ricerca Metrologica (INRIM), Torino, Italy

\section{T. Pfeifer}

RWTH Aachen University (RWTH), Aachen, Germany

M. Reader-Harris

National Engineering Lab, East Kilbride, Glasgow, UK

P.L. Regtien

University of Twente, Enschede, Netherlands

F. Righini

INRIM National Inst. Searches Metrologica, Torino, Italy

P.M.B. Silva Girāo

Portugal

K.D. Sommer

Physikalisch-Technische Bundesanstalt, Braunschweig, Germany

S. Tachi

University of Tokyo, Tokyo, Japan

J.C. Torres Guzmán

Centro Nacional de Metrologia - CENAM, Querétaro, Mexico

A. Weckenmann

Friedrich-Alexander-Universität Erlangen-Nürnberg, Erlangen, Germany 


\begin{tabular}{|c|c|c|c|c|c|}
\hline Keywords & Author name & Measurement & Volume & Issue & Pages \\
\hline
\end{tabular}

\section{Measurement}

SUPPORTS OPENACCESS

\section{Volume 129}

In progress (December 2018)

This issue is in progress but contains articles that are final and fully citable.
Download PDFs
^ Export
Expand all article previews

Estimation of oil reservoir parameters from temperature data for water injection Original research article

Pages 1-10

Yong-Le Nian, Wen-Long Cheng

Download PDF Article preview $\checkmark$

Radiofrequency planar surface coil for magnetic resonance: When the use of a circular wire gives a noticeable advantage with respect to a flat strip conductor?

Original research article

Pages 518-522

Giulio Giovannetti, Gianluigi Tiberi, Michela Tosetti, Agostino Monorchio, Nunzia Fontana

맘 Download PDF Article preview $\vee$

$\square$ Use of bi-level pulsed frequency-division excitation for improving blood oxygen saturation precision

Original research article

Pages 523-529

Shaoxiu Song, Fangfang Jiang, Liling Hao, Lisheng Xu, ... Ling Lin

문 Download PDF Article preview $\vee$

Analytical study of Roman red slip ware from ancient Tripolis (Denizli, Turkey)

Original research article

Pages 530-541

Barış Semiz, Bahadır Duman, Mehmet Ok

문 Download PDF Article preview $\vee$

$\square$ Liquid level sensor using fiber bundle

Original research article

Pages 542-547

Samian, A.H. Zaidan, M. Yasin, Pujiyanto, Supadi 


\title{
Measurement: Journal of the International Measurement Confederation
}

\author{
Country Netherlands \\ Subject Area and \\ Category \\ Engineering \\ Electrical and Electronic Engineering \\ Mathematics \\ Applied Mathematics \\ Statistics and Probability \\ Physics and Astronomy \\ Condensed Matter Physics \\ Instrumentation \\ Social Sciences \\ Education \\ Publisher \\ Elsevier BV \\ Publication type \\ Journals \\ ISSN \\ 02632241 \\ Coverage \\ 1983-ongoing \\ Scope Contributions are invited on all aspects of the research, development and applications of the \\ science and technology of measurement and instrumentation. Authors are encouraged to \\ submit novel material which could include results of research or experimental work, may deal \\ with practical developments related to plant or process, discuss new developments in \\ sensors and instrumentation, or relate to systems evaluation and modelling. Topics covered \\ include: General principles of measurement and instrumentation; Sensors and sensor \\ systems: design and evaluation; Data acquisition; Signal transmission; Processing and \\ evaluation; Data output; Systems aspects; Systems modelling and evaluation; Specific \\ applications in measurement and instrumentation. \\ How to publish in this journal \\ Join the conversation about this journal
}




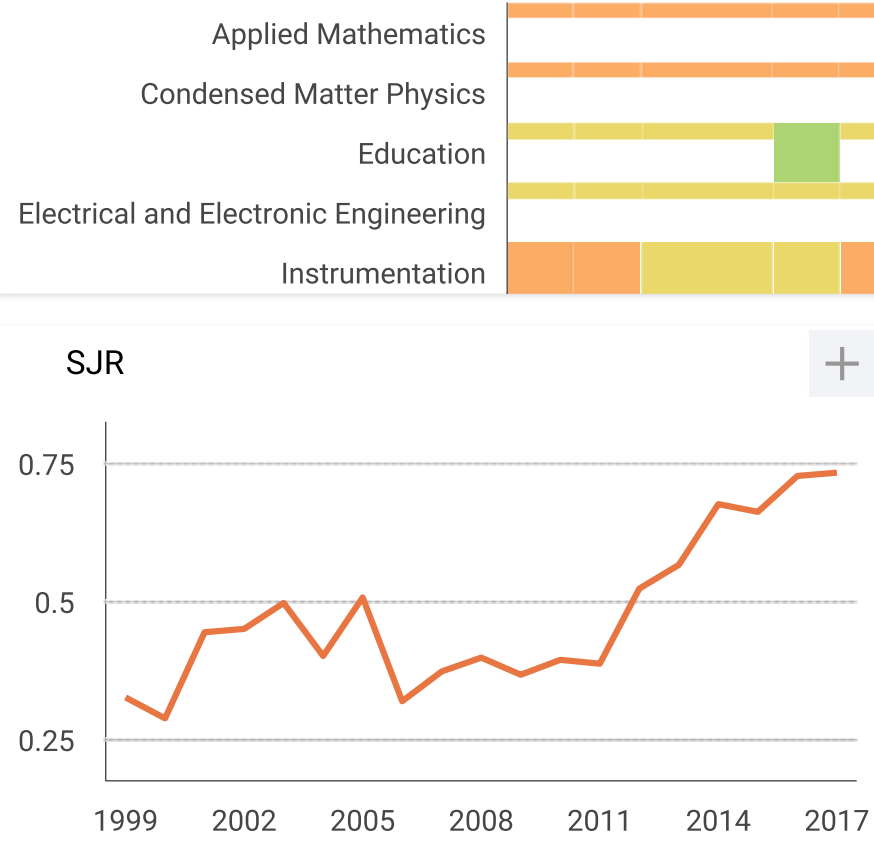

\section{Total Cites Self-Cites}

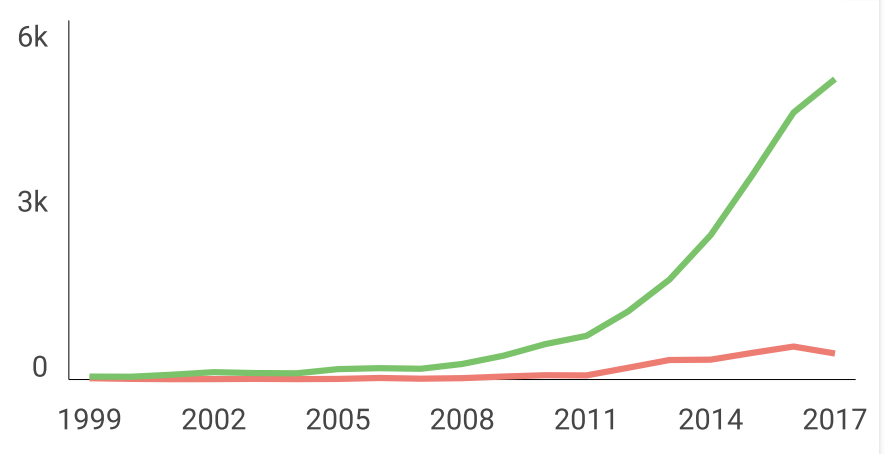

External Cites per Doc

Cites per Doc

Citations per document

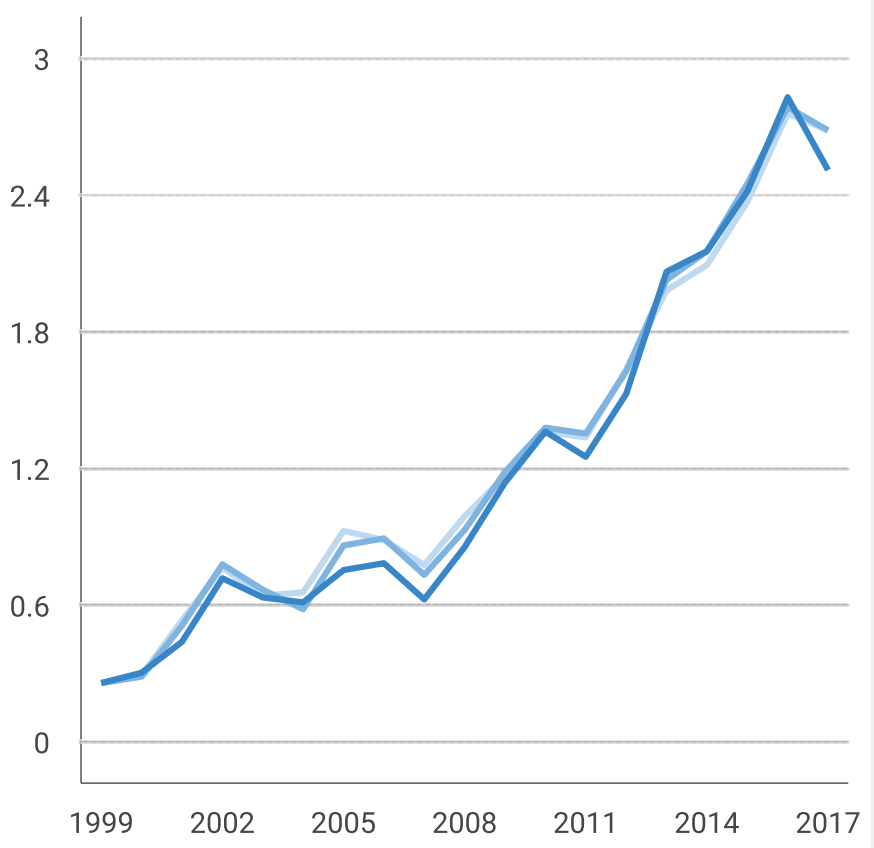

Cites / Doc. (4 years)

Cites / Doc. (3 years)

Cites / Doc. (2 years)

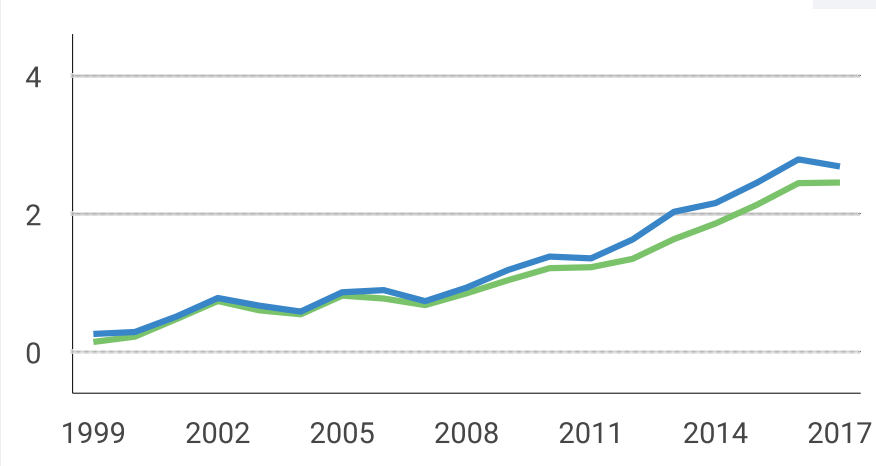

\% International Collaboration

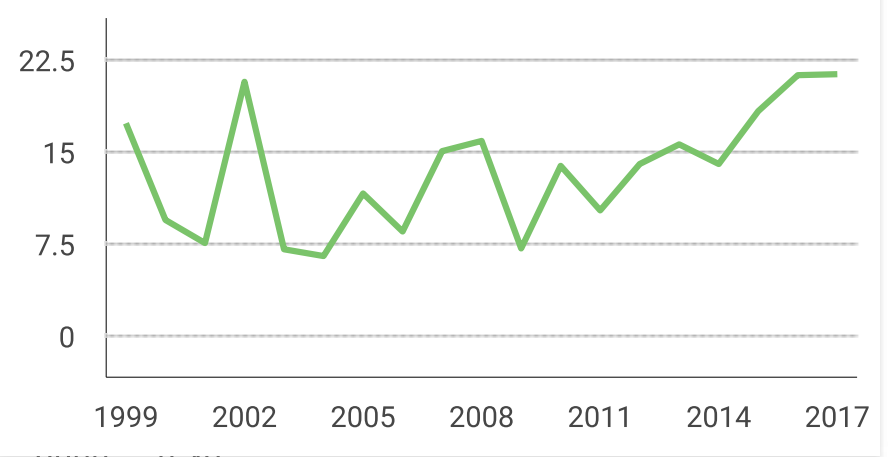

Citable documents Non-citable documents $\quad+$

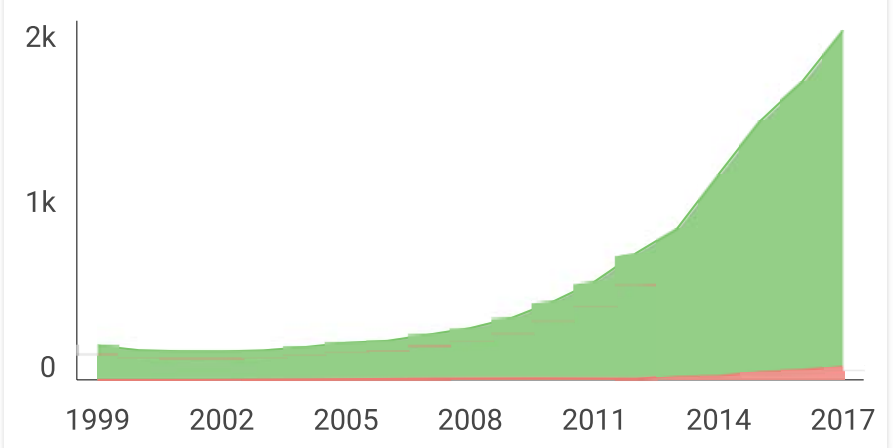

Cited documents Uncited documents

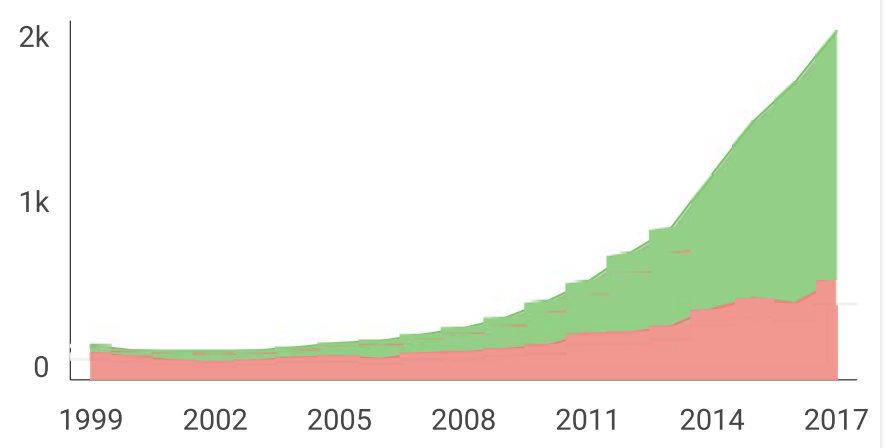

Show this widget in your own website 


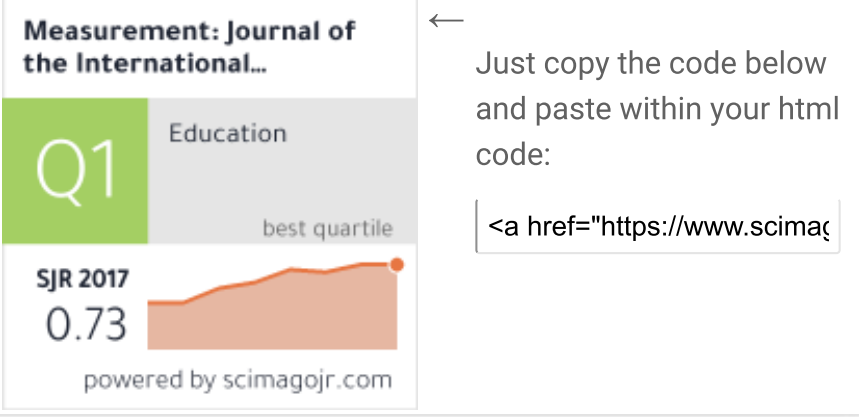

\section{Leave a comment}

Name

\section{Email}

(will not be published)

Saya bukan robot

Submit

The users of Scimago Journal \& Country Rank have the possibility to dialogue through comments linked to a specific journal. The purpose is to have a forum in which general doubts about the processes of publication in the journal, experiences and other issues derived from the publication of papers are resolved. For topics on particular articles, maintain the dialogue through the usual channels with your editor.
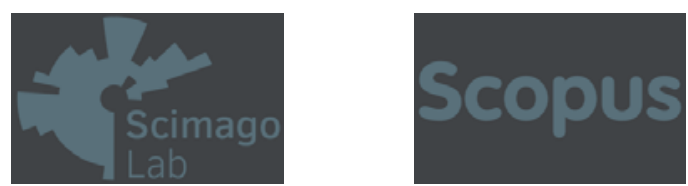


\title{
Liquid level sensor using fiber bundle
}

\author{
Samian*, A.H. Zaidan, M. Yasin, Pujiyanto, Supadi \\ Physics Department, Faculty of Science and Technology, Universitas Airlangga, 60115 Surabaya, Indonesia
}

\section{A R T I C L E I N F O}

\section{Keywords:}

Liquid level sensor

Fiber bundle

Displacement sensor

\begin{abstract}
A B S T R A C T
Liquid level sensors can be constructed using a pair of bundle probe based on fiber-optic displacement sensor. The working mechanism of the sensor is detecting displacement in the reflector which is attached to membrane (reflector displacement device or RDD) due to changes in the level of the liquid. Displacement of reflector is detected through changes in the intensity of reflected light from reflector into an optical fiber that serves as the recipient of the light. Other optical fiber acts as a transmitter of light. Three types of RDD was used in this experiment, namely RDD consisting of 1, 2, and 3 layers of the membrane. The best performance is shown by the sensor using RDD with 1 layer membrane. Sensor range, linear region, sensitivity, and resolution sensor generated each of $115 \mathrm{~cm}, 110 \mathrm{~cm}, 0.37 \mathrm{mV} / \mathrm{cm}$, and $1.45 \mathrm{~cm}$.
\end{abstract}

\section{Introduction}

Application of optical fiber as sensor can be based on the modulation of the intensity, wavelength, and phase. Application of optical fiber liquid level sensor has been done mostly based on the modulation of the intensity and wavelength. Optical fiber liquid level sensors based on wavelength modulation can use fiber Bragg grating [1], tapered chirped [2], and long period [3] which is connected with a buoy. The use of fiber optic interferometry with two photonic crystal fiber (PCF) that is placed on a trajectory of optical properties [4] has also been done. Sensor range for sensor based on wavelength modulation is not too wide (the order of $\mathrm{mm}$ ), but the resolution is good. For liquid level sensors with a wider range, intensity modulation is more likely to be used. Some examples of Optical fiber liquid level sensors based modulation intensity are using fiber optic connected with cone-shaped transparent elements [5], prism [6-8], and plastic optical fiber at its polished cladding [9] which acts as a sensor probe. The liquid level is detected when the sensor probe interacts with the liquid. Such interactions cause a change in the total reflected light intensity in the probe. Fiber coupler that has been used to detect the level of liquid (gasoline) is based on displacement sensor [10]. The sensing principle is to detect displacement of reflector, which is attached to the membrane (reflector displacement device), due to the change of liquid hydrostatic pressure. The displacement of reflector can be detected using fiber coupler from the change of optical power light reflected by the reflector. Liquid level sensors of this type have been developed until two levels so that the working area (linear region) doubled [11]. Other methods to detect liquid level are opto-fluidic techniques [12] and the transmission of optical signals on the surface of liquids [13]. Opto-fluidic technique is conducted through observation of a beam spot size of reflected surface of liquid. The optical signal broadcasting technique is carried out through an optical fiber and other optical fiber that serves as the receiver of the reflected signal from the surface of the liquid. Signals emitted or received by optical fiber is focused by a lens.

A pair bundle probe (fiber bundles consist of a pair) has been successfully to be used as a displacement sensor based on modulation of intensity. Displacement of reflector (mirror) detected through changes in the intensity of reflected light from the reflector go into one fiber that acts as the recipient of the beam of light, while other fiber serves as a transmitter of light to the reflector [14]. Based on working principle of liquid level sensor using fiber coupler as well as consider the ability of a pair bundle probes to detect displacement of reflector, then in this paper will be reported liquid level sensor that is constructed using a pair bundle probe.

\section{Sensors design and operating principle}

The working mechanism of liquid level sensor can be explained based on the schematic diagram of the sensor as shown in Fig. 1(a). The components of the sensor consist of a laser as light source, optical detectors, a pair bundle probes (each fiber serves as a transmitter and receiver light), and the tank as a container of liquid. At the base of the tank, there is a reflector displacement device (RDD), as shown in Fig. 1(b). RDD comprises a reflector (flat plate) which is glued together on the membrane. Reflectors will shift $\left(z^{\prime}\right)$ if the hydrostatic pressure changes $\left(P_{h}\right)$ due to a change in the liquid level $(h)$.

\footnotetext{
* Corresponding author.

E-mail address: samian@fst.unair.ac.id (Samian).
} 


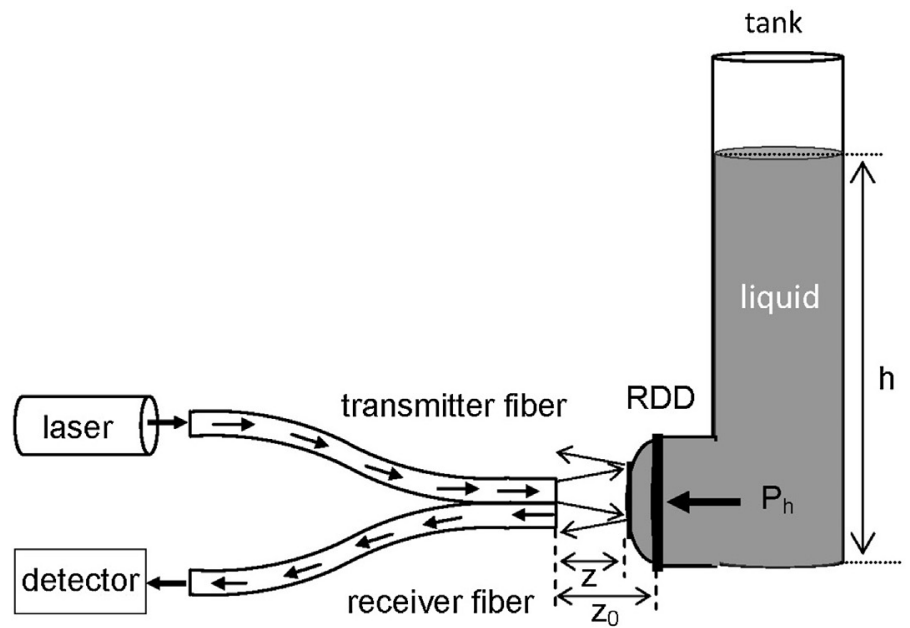

(a)

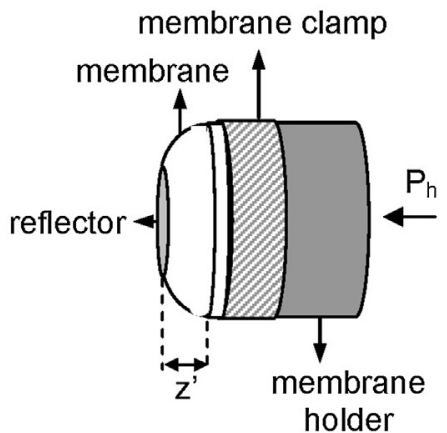

(b)

Fig. 1. The design of liquid level sensors using a pair bundle probe.

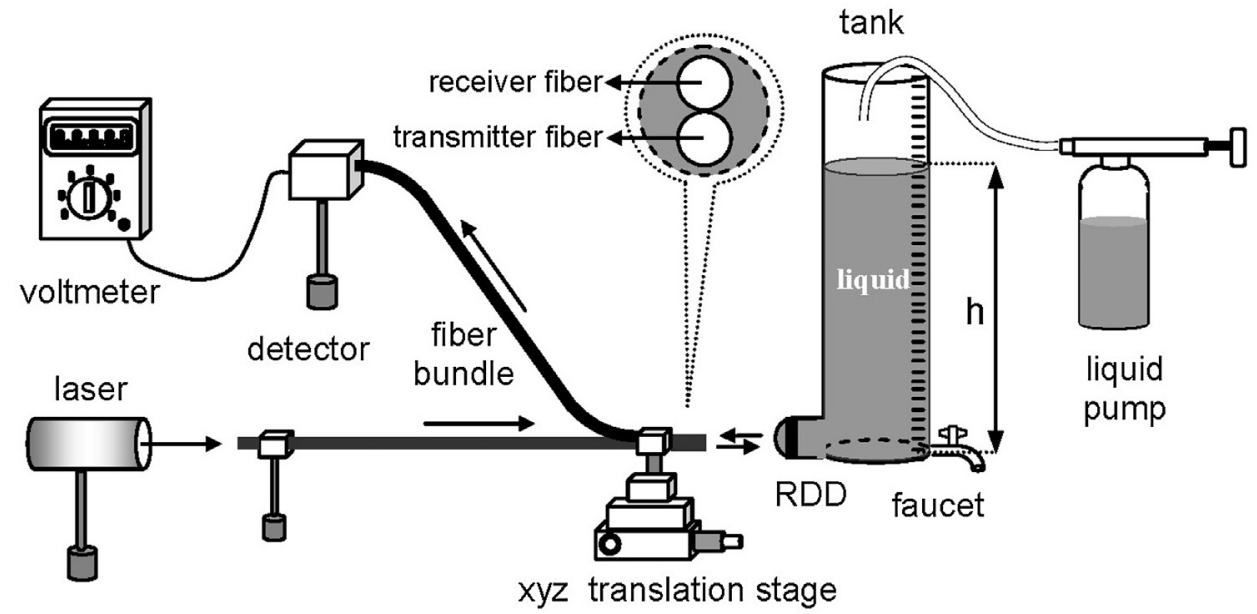

Fig. 2. Experimental set-up of liquid level sensor using a pair bundle probe.

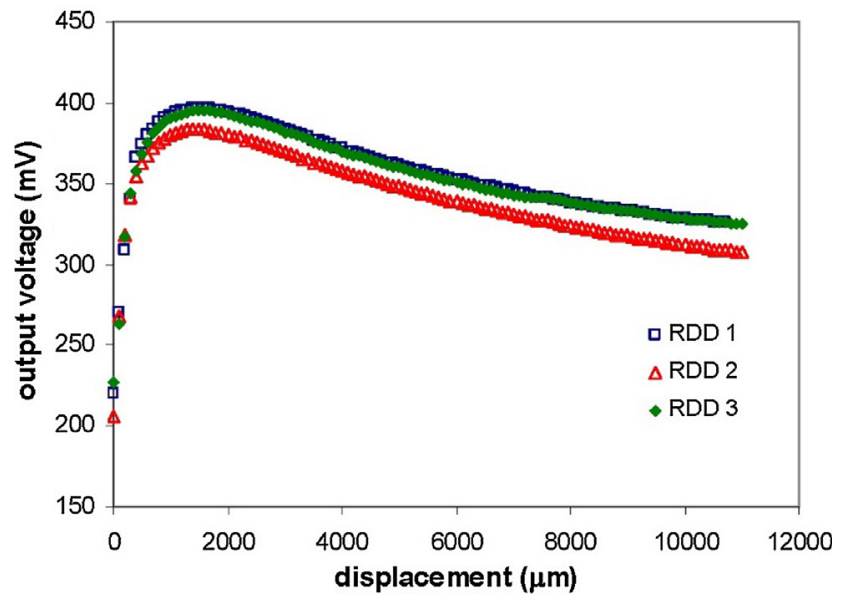

Fig. 3. The probe characteristic curve shifts to the reflector for all RDD used in the experiment.

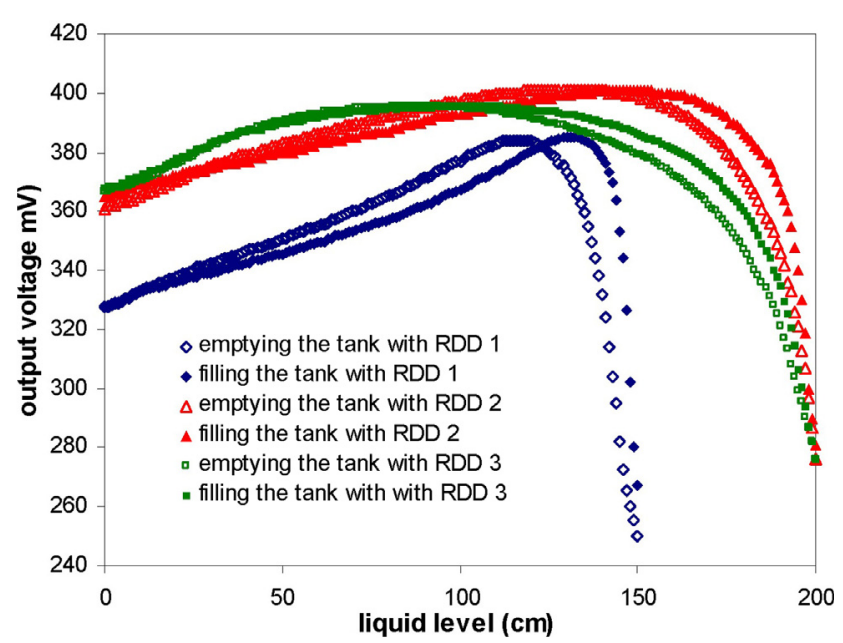

Fig. 4. Dynamic range (V versus $h$ ) for all RDD as result of scanning liquid level for to process of emptying and filling the tank. 
Table 1

Range of front slope and back slope are from liquid level scanning.

\begin{tabular}{|c|c|c|c|c|c|c|}
\hline \multirow[t]{2}{*}{ Area } & \multicolumn{2}{|l|}{ RDD 1} & \multicolumn{2}{|l|}{ RDD 2} & \multicolumn{2}{|l|}{ RDD 3} \\
\hline & Emptying & Filling & Emptying & Filling & Emptying & Filling \\
\hline $\begin{array}{l}\text { Range of } \\
\text { front } \\
\text { slope } \\
(\mathrm{cm})\end{array}$ & $0-116$ & $0-131$ & $0-130$ & $0-146$ & 0-90 & 0-104 \\
\hline $\begin{array}{r}\text { Range of } \\
\text { back } \\
\text { slope } \\
(\mathrm{cm})\end{array}$ & $117-150$ & $132-150$ & $131-200$ & $147-200$ & $91-200$ & $105-200$ \\
\hline
\end{tabular}

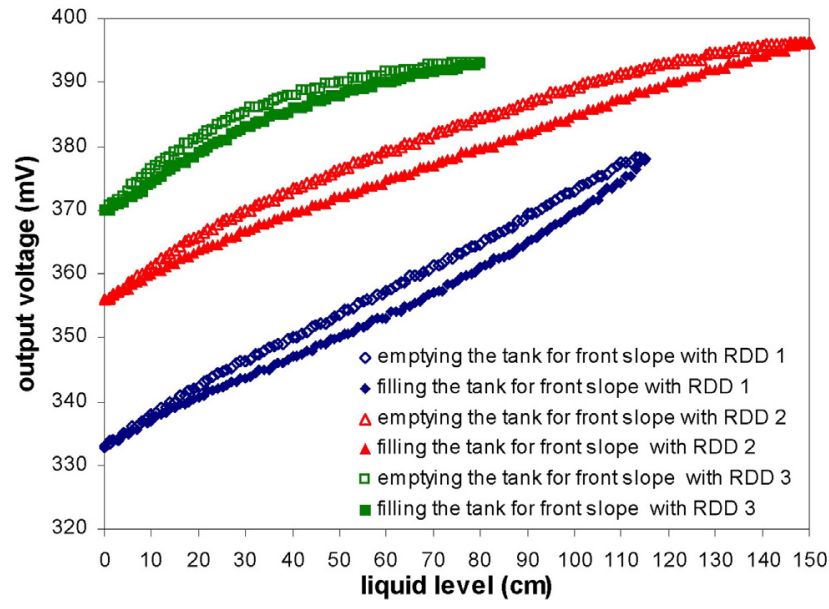

Fig. 5. Front slope curve of the liquid level sensor.

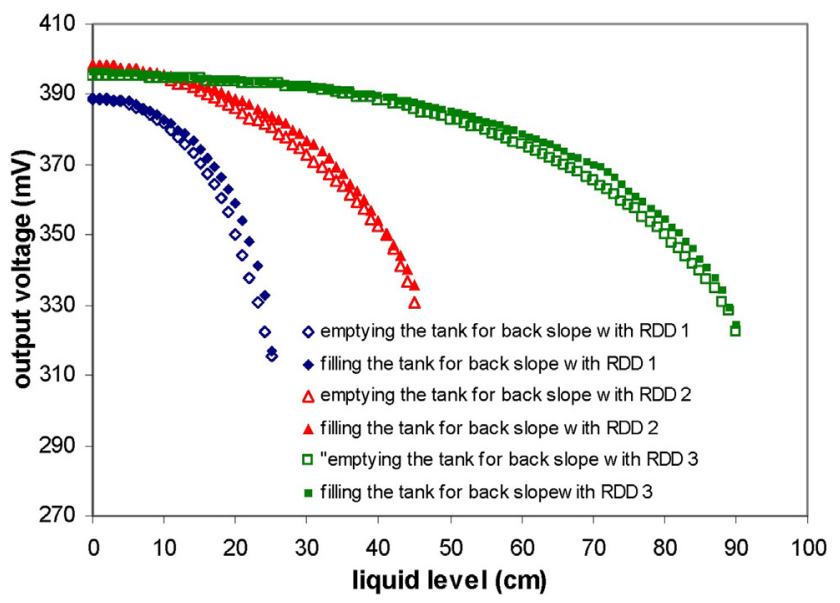

Fig. 6. Back slope curve of the liquid level sensor.

The detection principle of liquid level can be explained as follow: the light output of the transmitter fiber will be reflected by the reflector and received by the receiver fiber. The reflector will be shifting due to change in liquid level, as a result, the distance between the reflector toward the end of the fiber bundle that acts as a sensor probe (z) also will be changed. This change can be detected by the intensity of light received by the receiver fiber that is measured on optical detectors. Thus, the liquid level is detected through changes in light intensity that are measured on optical detectors (in this case changes the output voltage detectors).

The relationship between the output voltage $(V)$ with displacement of reflector against the probe $(z)$, in the area close to the probe $(z$ of about $1.7 \mathrm{~mm}$ ) produces the front slope, and the rest produces the back
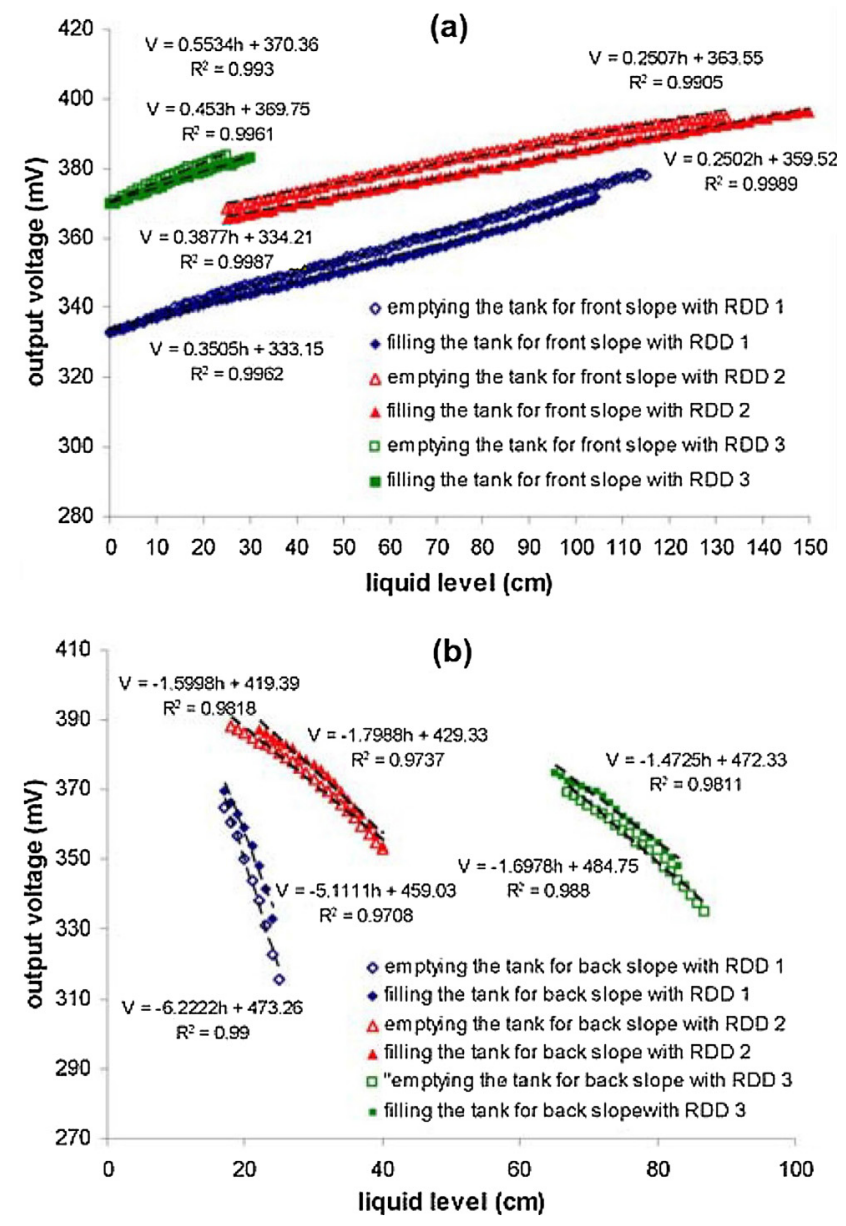

Fig. 7. Linear region of (a) front slope and (b) back slope.

slope [14]. Thus, the initial position of the probe against the reflector while the tank is empty $\left(z_{0}\right)$ will determine the characteristics of the sensor. For example, selected $z_{0}$ as far as the range of front slope $\left(z_{0}=1.7 \mathrm{~mm}\right)$, then the dynamic range of the sensor has only one slope. If selected $z_{0}>1.7 \mathrm{~mm}$ and range of liquids level being detected produces displacement in reflector more than $1.7 \mathrm{~mm}$, then the dynamic range of the sensor will produce two slopes (front slope and back slope). Therefore, the position of the $z_{0}$ will determine the range of the sensor.

\section{Experiment}

Schematic diagram of the experiment is shown in Fig. 2. The experimental set-up consists of a laser semiconductor (with $630 \mathrm{~nm}$ wavelength and a 10-mW power output), silicon photodetector (818 SL), a pair bundle probes (from plastic material with $1 \mathrm{~mm}$ diameter and $2 \mathrm{~m}$ length) as a sensor probe, voltmeter (digital multimeter), xyz translation stage used to shift the probe sensor, manual liquid pump is used to pump liquid into the tank, cylindrical scaled water tank which is made of glass (with a diameter of $6 \mathrm{~cm}$ and $200 \mathrm{~cm}$ in length). In the bottom of the water tank, there are a faucet that is used for emptying the tank and reflector displacement device (RDD) consist of reflector which is made of aluminum (with $10 \mathrm{~mm}$ in diameter and thickness $0.20 \mathrm{~mm}$ ), membrane (nitrile polymer) with $0.08 \mathrm{~mm}$ thickness (the elastic modulus is not known), and cylindrical membrane holder which is made of brass with $18 \mathrm{~mm}$ outer diameter and inner diameter of $15 \mathrm{~mm}$. Reflector is attached to membrane using epoxy glue and membrane itself behaves like a spring. Three types of RDD used in this experiment i.e. RDD 1, RDD 2, and RDD 3 each consisting of 1, 2, and 3 layers membrane. In this experiment, the liquid used is water. 

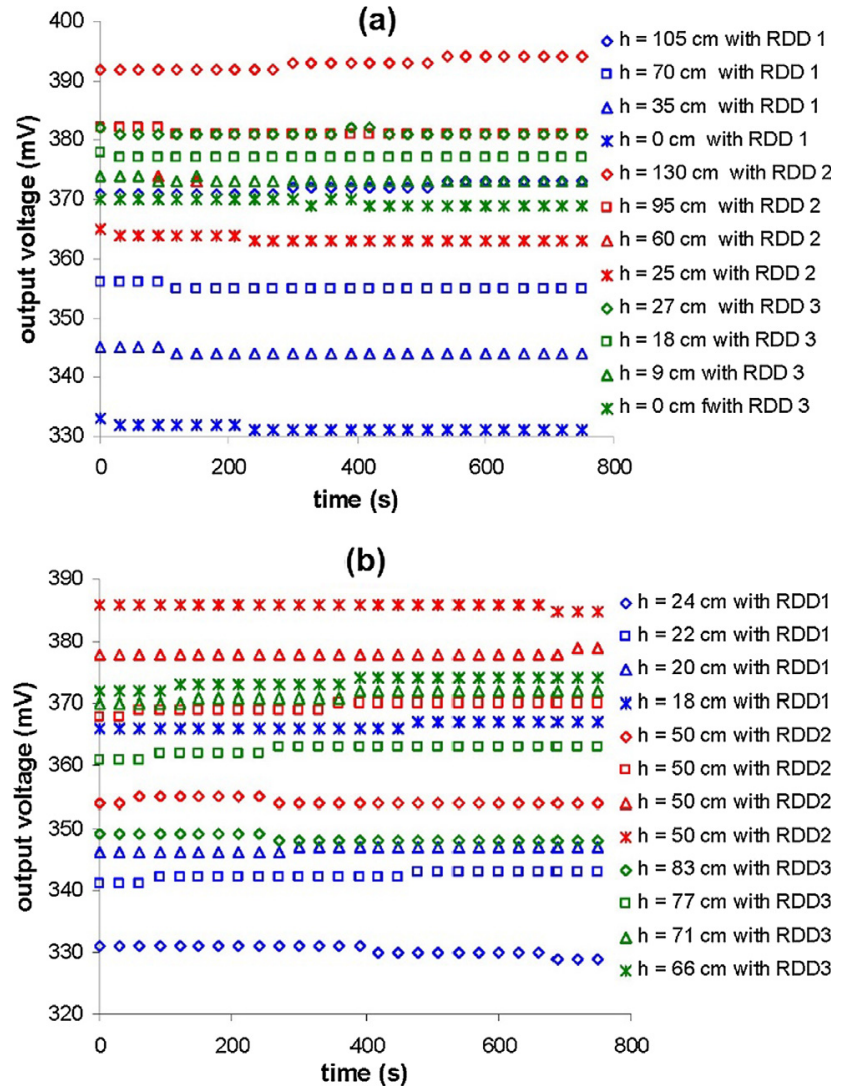

Fig. 8. Sensor stability curve for the area of (a) front slope and (b) back slope.

The first step of the experiment is to characterize the displacement of sensor probe against the reflector. The data were taken by placing a sensor probe located at the center and coincide with reflector RDD 1 (1 membrane layers) when the tank is empty. The recording of output voltage performed each probe shifted backward of $100 \mathrm{~mm}$. Probe Shifting was done using xyz translation stage. The second step is to characterize the liquid level sensor on the emptying process of the tank. The second step begins by filling the tank with liquid as high as $150 \mathrm{~cm}$, then place the probe coincide with reflectors RDD $1(z=0 \mathrm{~mm})$. The liquid level limit was selected at $150 \mathrm{~cm}$ due to the maximum ability of 1 layer membrane to withstand hydrostatic pressure (liquid level more than $150 \mathrm{~cm}$ can deform 1 membrane layer). Furthermore, the recording of output voltage is performed every liquid level is lowered by $1 \mathrm{~cm}$. After tank is empty, experiment proceeds to third step i.e. characterization of sensors when filling the tank with liquid. The third step is carried out by means of filling the tank with liquid using pumps. Each increase of $1 \mathrm{~cm}$ liquid level, recording of output voltage detector is performed. The recording was performed until the maximum level of the liquid in the tank is the same as the emptying process. The procedure is repeated three times. The procedure then repeated for RDD 2 ( 2 layers membrane) and RDD 3 (3 layers membrane). Because RDD 2 ability to withstand hydrostatic pressure is better than RDD 1, then the maximum level of the liquid used in the experiment for RDD 2 and RDD 3 is $200 \mathrm{~cm}$.

The second and third step is scanning the liquid level, it was done to get the dynamic range of the relationship between the output voltage against the liquid level. Due to the displacement of the reflectors exceeded $1.7 \mathrm{~mm}$, then the dynamic range has front slope and back slope. So, the front slope and back slope will be selected from the second and third step results. The fourth step is characterization of liquid level sensor respectively on the front slope and back slope. The final step is to test the stability of the sensor. The test was performed by measuring the output voltage in a span of $30 \mathrm{~s}$ over the $750 \mathrm{~s}$. Stability test conducted on the working area of the sensor range which is divided into four-point liquid level.

\section{Result and discussion}

The characteristics of the probe displacement against all reflector RDD can be known through a voltage output as a function of the probe displacement. The results are shown by the curve in Fig. 3. From Fig. 3, it appears that the characteristics of the probe displacement against the reflector for all RDD are almost the same. This means that the reflector coincides on all RDD is almost identical. The curve in Fig. 3 has front slope on the displacement range of $1.5 \mathrm{~mm}$ and rest of the displacement range $(9.5 \mathrm{~mm})$ is the back slope. The occurrence of two slopes (front slope and back slope) on the dynamics range of displacement profile can be reviewed from sensor geometry. The sensor probe consists of two optical fibers adjacent to each other. An optical fiber acts as a transmitter and other optical fibers act as receivers. When the probe position coincides with the reflector, the output light from the transmitter fiber reflected off by the reflector, all goes back to the transmitter fiber. Thus no light enters the receiver fiber. The light received by the receiver fiber is read in the form of the output voltage of the detector. As the sensor probe begins to shift away from the reflector, a small portion of the reflected (reflected) light of the reflector begins to enter the receiver fiber. The larger the probe shift, the wider the reflected light beam of the reflector coming into the receiver fiber receiver. At a displacement of $1.5 \mathrm{~mm}$, the width of the fiber receiver area is filled with reflective light beam from the reflector so that the maximum incoming light intensity (front slope) occurs. If the displacement is increased, then the reflected light beam from the reflector will expand. On the other hand, the fiber cross section area is fixed. Thus the intensity of light entering the receiver fiber decreases as the displacement is increased. The condition produces a back slope (1.5-11 mm shift).

The dynamic range of the sensor is known from scanning the liquid level sensor which produces an output voltage $(V)$ in response to changes in the liquid level $(h)$. For all RDD, the scanning results are shown in Fig. 4. Dynamic range for RDD 1 is $150 \mathrm{~cm}$, while RDD 2 and RDD 3 have equivalent result i.e. $200 \mathrm{~cm}$.

The curve in Fig. 4 shows that the front slope much more shallow than the back slope (inverted compared with the results of displacement characterization). This occurs because when the water level is maximum, the hydrostatic pressure also maximum so that the reflector position close to the sensor probe. The results also show that the dynamic range between filling and emptying process (hysteresis) is not the same. The difference is due to the nature of membrane relaxation after stress. The larger the membrane under pressure, the longer the relaxation time. It can be seen on the back slope of the curve in Fig. 4 . The range of front slope and back slope for all RRD is shown in Table 1. The $z_{0}$ value for RDD 1 , RDD 2, and RDD 3 respectively are $7.7 \mathrm{~mm}$, $4.77 \mathrm{~mm}$, and $3.95 \mathrm{~mm}$.

In dynamic range, there is a voltage output whose value is the same but for two different levels (located on the front slope and back slope). Therefore, the dynamic range is not the sensor range desired. To get the sensor range, we have conducted experiments with a limited range of liquid levels. Limit of level liquid used is based on the data in Table 1 to produce a front or back slope only. To get the front slope only, $z_{0}$ is in the same position as when the liquid level scanning is performed and maximum limit of liquid level is the level indicated in Table 1 . To get back slope only, the sensor probe is made coincident with the reflector when the water level has reached the limit of the range of local produce back slope. Data retrieval is performed three times.

The result of experiment to produce front slope of the sensor is shown in Fig. 5. From the curve in Fig. 5, it is known that the range of detection for RDD 1, RDD 2, and RDD 3 is respectively $115 \mathrm{~cm}, 150 \mathrm{~cm}$, and $80 \mathrm{~cm}$. The distance between the reflector to the probe $(z)$ of the at the maximum level for RDD 1, RDD 2, and RDD 3 is respectively $1.7 \mathrm{~mm}, 0.8 \mathrm{~mm}$, and $1.5 \mathrm{~mm}$. The average difference of output voltage 
Table 2

Characteristics of the liquid level sensor using a pair bundle probe.

\begin{tabular}{|c|c|c|c|c|c|c|c|c|c|c|c|c|}
\hline \multirow[t]{3}{*}{ Parameter } & \multicolumn{6}{|l|}{ Front slope } & \multicolumn{6}{|l|}{ Back slope } \\
\hline & \multicolumn{2}{|l|}{ RDD 1} & \multicolumn{2}{|l|}{ RDD 2} & \multicolumn{2}{|l|}{ RDD 3} & \multicolumn{2}{|l|}{ RDD 1} & \multicolumn{2}{|l|}{ RDD 2} & \multicolumn{2}{|l|}{ RDD 3} \\
\hline & Emptying & Filling & Emptying & Filling & Emptying & Filling & Emptying & Filling & Emptying & Filling & Emptying & Filling \\
\hline Sensor range $(\mathrm{cm})$ & 115 & 115 & 150 & 150 & 80 & 80 & 25 & 25 & 45 & 45 & 90 & 90 \\
\hline Linear region $(\mathrm{cm})$ & $0-115$ & $0-104$ & 25-132 & $25-150$ & $0-25$ & $0-30$ & $17-25$ & $17-24$ & $18-40$ & $22-40$ & $67-87$ & $65-83$ \\
\hline Sensitivity $(\mathrm{mV} / \mathrm{cm})$ & 0.39 & 0.35 & 0.25 & 0.25 & 0.55 & 0.45 & 6.22 & 5.11 & 1.6 & 1.8 & 1.7 & 1.47 \\
\hline Resolution (cm) & 1.4 & 1.5 & 2.1 & 2.1 & 0.6 & 0.8 & 0.1 & 0.1 & 0.3 & 0.2 & 0.4 & 0.5 \\
\hline
\end{tabular}

Table 3

The comparison of performance between optical level sensors.

\begin{tabular}{|c|c|c|c|c|c|}
\hline No. & Method & Sensor range $(\mathrm{m})$ & Linear region $(\mathrm{m})$ & Resolution $(\mathrm{cm})$ & Ref. \\
\hline 1. & Fiber Bragg Grating & 0.36 & 0.4 & 6 & {$[1]$} \\
\hline 2. & Tapered chirped fiber grating & 0.05 & 0.05 & - & {$[2]$} \\
\hline 3. & Long-period Fiber Bragg Grating & 1 & - & 10 & [3] \\
\hline 4. & fiber interferometer using PCF & 0.05 & 0.05 & 0.5 & [4] \\
\hline 5. & Fiber optic-prism & 0.014 & - & 0.1 & [7] \\
\hline 6. & Plastic optical fiber polished & 2.7 (discontinue) & - & - & [9] \\
\hline 7. & One output port fiber coupler & 1.8 & 0.4 & 0.6 & [10] \\
\hline 8. & Two output port fiber coupler & 1 & 0.7 & 0.4 & [11] \\
\hline 9. & Opto-fluidic & 0.75 & - & 1.7 & [12] \\
\hline 10. & Optical signal broadcasting & 2 & - & - & [13] \\
\hline 11. & A pair bundle probe (our paper) & 1.15 & 1.1 & 1.4 & - \\
\hline
\end{tabular}

at the same level for the process of filling and emptying for RDD 1, RDD 2 , and RDD 3 is respectively $0.8 \%, 0.85 \%$, and $0.40 \%$. The largest measurement errors on RDD 1, RDD 2, and RDD 3 respectively are $0.9 \mathrm{mV}(0.4 \%), 1 \mathrm{mV}(0.25 \%)$, and $0.57, \mathrm{mV}(0.15 \%)$. Apparently, the thicker the membrane, the smaller the measurement error.

For the back slope, the result is shown by the curves in Fig. 6 . The average difference of output voltage at the same level for process of filling and emptying for RDD 1 RDD 2, and RDD 3 is respectively $0.8 \%$, $0.6 \%$, and $0.4 \%$. The largest measurement errors on RDD 1 , RDD 2 , and RDD 3 respectively are $0.7 \mathrm{mV}(0.3 \%), 0.58 \mathrm{mV}(0.16 \%)$, and $0.57, \mathrm{mV}$ $(0.15 \%)$. The $z_{0}$ value for RDD $1, \operatorname{RDD} 2$, and RDD 3 respectively are $5.53 \mathrm{~mm}, 2.7 \mathrm{~mm}$, and $2.45 \mathrm{~mm}$.

The back slope curve in Fig. 6, has a smaller slope than the curves in Fig. 4. This occurs because of the elasticity of the membrane decreases when the membrane gets smaller hydrostatic pressure so that the membrane displacement range depends on the magnitude of the hydrostatic pressure (liquid level) and not on the range of liquid level. That means reflector displacement is not linear against the hydrostatic pressure.

The linear region which is the working area of the sensor for front and back slope is shown in Fig. 7. The lowest level of linearity for the region is $99.5 \%$ for front slope and $98.5 \%$ for back slope. The slope in the linear region is the value of sensor sensitivity. The sensitivity of the sensor in the front slope area is smaller than the area of the back slope, but sensor range of front slope is a much larger than the back slope, except for the RDD 3. This happens due to limited level of the tank used in this experiment. In our opinion, dynamic range of sensor using RDD 3 could be even greater because membrane of RDD 3 is thicker than RDD 1 and RDD 2 so that it able to withstand the water pressure with an altitude higher than $200 \mathrm{~cm}$.

Sensor stability test performed by selecting four points of the water level in the range of the sensor linear region. The results are shown in the curve of Fig. 8(a) and (b) respectively for the linear front slope and back slope. The largest standard deviation value in the linear region of front slope of RDD 1, RDD 2, and RDD 3 respectively is $0.54 \mathrm{mV}$, $0.54 \mathrm{mV}$, and $0.35 \mathrm{mV}$. While on the linear region of back slope, the largest standard deviation value for RDD 1, RDD 2, and RDD 3 respectively is $0.59 \mathrm{mV}, 0.42 \mathrm{mV}$, and $0.68 \mathrm{mV}$. From these results, it can be concluded that the stability of the sensor is good. The largest standard deviation value if divided by the value of the sensitivity of the sensor value will be the resolution of the sensor.

The sensor performance can be known through parameters such as sensor range, the range of the linear region, sensitivity, and resolution. Overall, the performance of the sensor both for front slope area and the back slope area is shown in Table 2. Based on the value of the parameters in Table 2, it is known that the best sensor performance in our opinion is a sensor that uses RDD 1 on the front slope area. The reason is because the working area of the sensor (linear region) starts from $0 \mathrm{~cm}$. Although its range is smaller than the sensor using RDD 2, but the sensitivity and the resolution are better. For sensors using RDD 3, the actual characteristic cannot be observed due to the limited level of the tank used in the experiments. For sensors with a range of back slope area, although the sensitivity and resolution far better than the sensors with a range of front slope area, but the range and the area of work is lower. Excluding the parameter values for the use of RDD 3, the thicker the membrane is used, the larger the range and linear region sensors (the mean value between emptying and filling) are generated. Conversely, the thinner the membrane is used, the greater the membrane elasticity. Increase in membrane elasticity causes increase in sensor sensitivity. The impact of increase in sensitivity of the sensor is the smaller the value of the sensor resolution. This occurs due to the proposed sensor mechanism uses the hydrostatic pressure principle to detect the level of the liquid. However, each sensor either front slope or back slope can be used in accordance with needs, not only to detect the water but can also be used for gasoline $[10,11]$

The performance of the liquid level sensor using the optical fiber as a whole is shown in Table 3. Methods 1-4 is based on wavelength modulation. Methods 5-8 and 11 are based on intensity modulation. Methods 9 and 10 use opto-fluodic and Optical signal broadcasting respectively. Methods 7, 8, and 11 use the same working principle with our sensor that is based on optical fiber displacement sensors by utilizing hydrostatic pressure. When compared to results in methods 7 and 8 , it appears that the working area of the sensor in our experiment is larger. 


\section{Conclusions}

A pair bundle probes can be applied as a liquid level sensor based on displacement sensor. Utilization of hydrostatic pressure changes due to changes in liquid levels to shift the reflector is the basic working mechanism of the sensor. The experimental results show the use of 1 layer membrane as reflector displacement device produces the best performance sensor. Sensor range, working area (linear region), sensitivity, and resolution of the sensor with 1 layer membrane respectively are $115 \mathrm{~cm}, 110 \mathrm{~cm}, 0.37 \mathrm{mV} / \mathrm{cm}$, and $1.45 \mathrm{~cm}$.

\section{References}

[1] Kyung-Rak Sohn, Joon-Hwan Shim, Liquid-level monitoring sensor systems using fiber Bragg grating embedded in cantilever, Sens. Actuators A 152 (2009) 248-251.

[2] D.O.N.G. XiaoWei, L.I.U. WenKai, Z.H.A.O. RuiFeng, Liquid-level sensor based on tapered chirped fiber grating, Sci. China Tech. Sci. 56 (2) (2013) 471-474.

[3] Jian-Neng Wang, Ching-Ying Luo, Long-period fiber grating sensors for the measurement of liquid level and fluid-flow velocity, Sensors 12 (2012) 4578-4593.

[4] Xinpu Zhang, Wei Peng, Zigeng Liu, Zhenfeng Gong, Fiber optic liquid level sensor based on integration of lever principle and optical interferometry, IEEE Photon. 6
(2) (2014) 6801108.

[5] Pekka Raatikainen, Ivan Kassamakov, Roumen Kakanakov, Mauri Luukkala, Fiberoptic liquid-level sensor, Sens. Actuators A 58 (1997) 93-97.

[6] Chengning Yang, Shiping Chen, Guoguang Yang, Fiber optical liquid level sensor under cryogenic environment, Sens. Actuators A 94 (2001) 69-75.

[7] Hossein Golnabi, Design and operation of a fiber optic sensor for liquid level detection, Opt. Lasers Eng. 41 (2004) 801-812.

[8] G. Onorato, G. Persichetti, I.A. Grimaldi, G. Testa, R. Bernini, Optical fiber fuel level sensor for aeronautical applications, Sens. Actuators A 260 (2017) 1-9.

[9] M. Lomer, J. Arrue, C. Jauregui, P. Aiestaran, J. Zubia, J.M. Lopez-Higuera, Lateral polishing of bends in plastic optical fibres applied to a multipoint liquid-level measurement sensor, Sens. Actuators A 137 (2007) 68-73.

[10] Samian, G.Y.Y. Yhosep, A.H. Zaidan, Herlik Wibowo, Gasoline level sensor based on displacement sensor using fiber coupler, Measurement 58 (2014) 342-348.

[11] Samian, Y.G.Y. Yhuwana, A.H. Zaidan, Supadi, Sujito, Application of two-output port fiber coupler as gasoline level sensor, Measurement 85 (2016) 80-87.

[12] Syed Azer Reza, Nabeel A. Riza, Agile lensing-based non-contact liquid level optical sensor for extreme environments, Optics Commun 283 (2010) 3391-3397.

[13] C. Vázquez, A.B. Gonzalo, S. Vargas, J. Montalvo, Multi-sensor system using plastic optical fibers for intrinsically safe level measurements, Sens. Actuators A 116 (2004) 22-32.

[14] M. Yasin, S.W. Harun, H.A. Abdul-Rashid, Karyono Kusminarto, A.H. Zaidan, H. Ahmad, Performance of optical displacement sensor using a pair type bundled fiber from a theoretical and experimental perspective, J. Optoelectron. Adv. Mater. Rapid Commun. 1 (11) (2007) 549-553. 